\title{
The assessment of impact of the crack size on the fracture load of a cylindrical element
}

\author{
Petr Kulakov $^{l}$, Bulat Kutlubulatov ${ }^{1}$, Aleksey Rubtsov ${ }^{l}$, Zinur Mukhametzyanov ${ }^{l}$ and Vitaliy \\ Afanasenko $^{l}$ \\ ${ }^{1}$ Ufa State Petroleum Technological University, Ufa, 450064, Russia
}

\begin{abstract}
Fracture mechanics is an important area in the study of materials and structures. In this paper, we will consider the use of tools that are available in this area using the Ansys program. The calculation of the strength of the structure, the assessment from the resource and reliability are carried out in many cases, taking into account the possibility of the presence of technological or operational cracks in them. Loss of constructional integrity can occur for many reasons: selection of inappropriate materials, inadequate design, defects in manufacturing, excess service life, environmental conditions, etc. It became necessary to develop a separate area of knowledge, the invention of separate methods dedicated to the structure with a crack. This is because the design of structural components has traditionally been based on material strength. This approach does not imply an increased stress level due to the existing crack, which can lead to incorrect results.
\end{abstract}

\section{Keywords}

J-integral, SMART Crack Growth, Crack Modeling Tools

\section{Introduction}

Fracture mechanics is a branch of mechanics that studies structural materials and their ability to resist fracture under the influence of external forces in the presence of fatigue cracks and various technological operational defects. Accordingly, Fracture analysis assumes the presence of an initial crack [1-5].

The fracture mechanics approach takes into account the presence of cracks or defects in the structure. Here, defect size is very important, and fracture toughness (fracture toughness) replaces strength (ultimate stress) as an appropriate parameter.

Typical fracture mechanics parameters describe either the rate of energy release or the amplitude of stress fields at the crack tip.

The following parameters are widely used in fracture mechanics:

- J-integral

- $\quad$ Energy-Release Rate (energy release rate)

- Stress-Intensity Factor (Stress intensity factor)

- T-stress

- Material Force (Configuration forces)

- $\quad$-integral.

The most commonly used parameters of fracture mechanics are the stress intensity factor $(\mathrm{K})$, the Jintegral, and the velocity (intensity) of the released elastic energy (G).

III International Workshop on Modeling, Information Processing and Computing (MIP: Computing-2021), May 28, 2021, Krasnoyarsk, Russia

EMAIL: kulakov.p.a@mail.ru (Petr Kulakov); bulat.kutlubulatov@bk.ru (Bulat Kutlubulatov); sunset202@mail.ru (Aleksey Rubtsov); zinur1966@mail.ru (Zinur Mukhametzyanov); afanasenko.v.g@yandex.ru (Vitaliy Afanasenko)

(c) (1) 2021 Copyright for this paper by its authors.

Use permitted under Creative Commons License Attribution 4.0 International (CC BY 4.0).

CEUR Workshop Proceedings (CEUR-WS.org) 
The other three parameters perform more specific functions. For example, the $\mathrm{C}$ integral is used instead of the $\mathrm{J}$ integral in problems with creep, and configurational forces are used in structures made of composite materials. T-stresses are used to estimate the size of the plastic deformation zone near the crack (as well as to assess the stress-strain state and the stability of the possible path of crack propagation).

The main parameter used in linear fracture mechanics is the fracture intensity factor $\mathrm{Kc}$. It is a scale factor used to describe the increase in applied stress at the tip of a fracture of known size and shape. The stress intensity value at which the crack starts to propagate is called the critical stress intensity factor or toughness [6-10].

\section{Experimental technique}

In general, the process of solving the problem of fracture mechanics can be divided into 2 large stages:

- calculation of fracture mechanics parameters. At the initial stage, one or another parameter is calculated (mainly the stress intensity factor) and its comparison with the critical value of the material makes it possible to establish whether the crack will grow under the given loads. In the overwhelming majority of cases, this stage is limited.

- modelling of the crack propagation process. Ansys Mecanical implements a number of techniques that allow simulating, among other things, the growth of a crack when the value of the parameter exceeds the critical level. In addition, there is a specific approach to modelling fatigue crack growth.

Before the first stage, there may also be a preliminary design (estimate) of the structure without a crack to determine the location

The calculation of fracture mechanics parameters is carried out by the CINT method, which performs independent calculations along some integration contours.

The advantage of this approach is that it does not require the creation of singular finite elements in the form of a crack.

Since, by definition, $\mathrm{J}$ of the integral does not depend on the path of integration, the result of the numerical calculation should converge to a certain value after the first few contours.

Thus, the structure must have a crack with a mesh of a certain size (presence of integration contours). Depending on the selected tool, the crack can be created automatically at the mesh level (Figure 1) or manually at the geometry level.

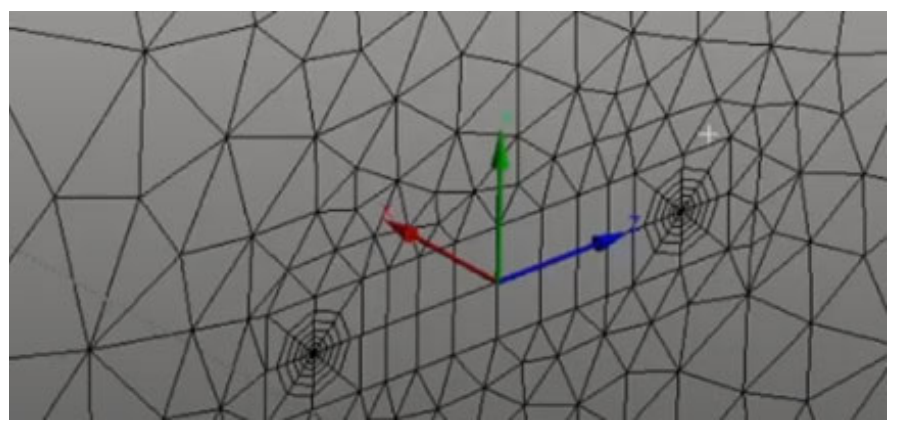

Figure 1. Example of a crack mesh

Depending on the selected tool, the mesh can consist of either hex or tetra elements. A special coordinate system must also be created for the crack: the $\mathrm{X}$ axis is normal to the body surface, the $\mathrm{Z}$ axis is along the crack edge, and the $\mathrm{Y}$ axis is perpendicular to the crack edge. Modelling (growth or propagation, passage) of a crack is a phenomenon in which two surfaces of a crack are separated, or the material is successively damaged under external loading.

\subsection{Crack Modeling Tools}


The original crack is required to solve the fracture mechanics. There are three tools to create it in Ansys Mecanical in the Fracture branch (Figure 2):

- Semi-Elliptical Crack.

- Freeform Crack Arbitary Crack.

- Crack created by hand during the Pre-Meshed Crack geometry creation stage.

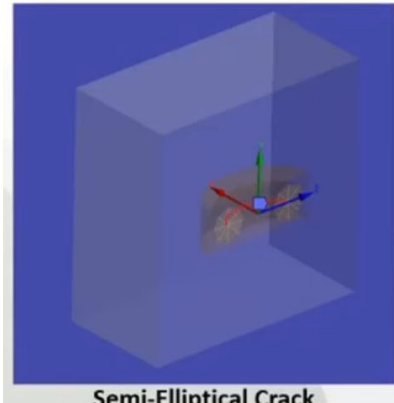

Semi-Elliptical Crack

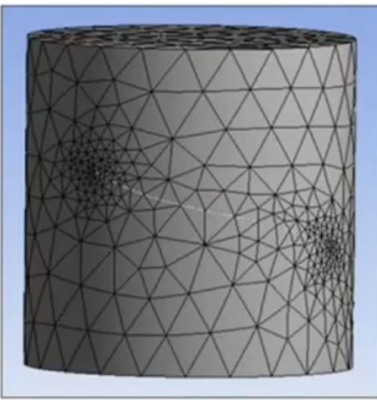

Arbitrary Crack

Figure 2. Methods for specifying a crack

\subsection{Semi-elliptical crack}

The Semi-Elliptical Crack tool allows you to create a semi-elliptical model.

All characteristic dimensions of the elliptical shape are directly set in the properties window of the crack object and completely define it (Figure 3).
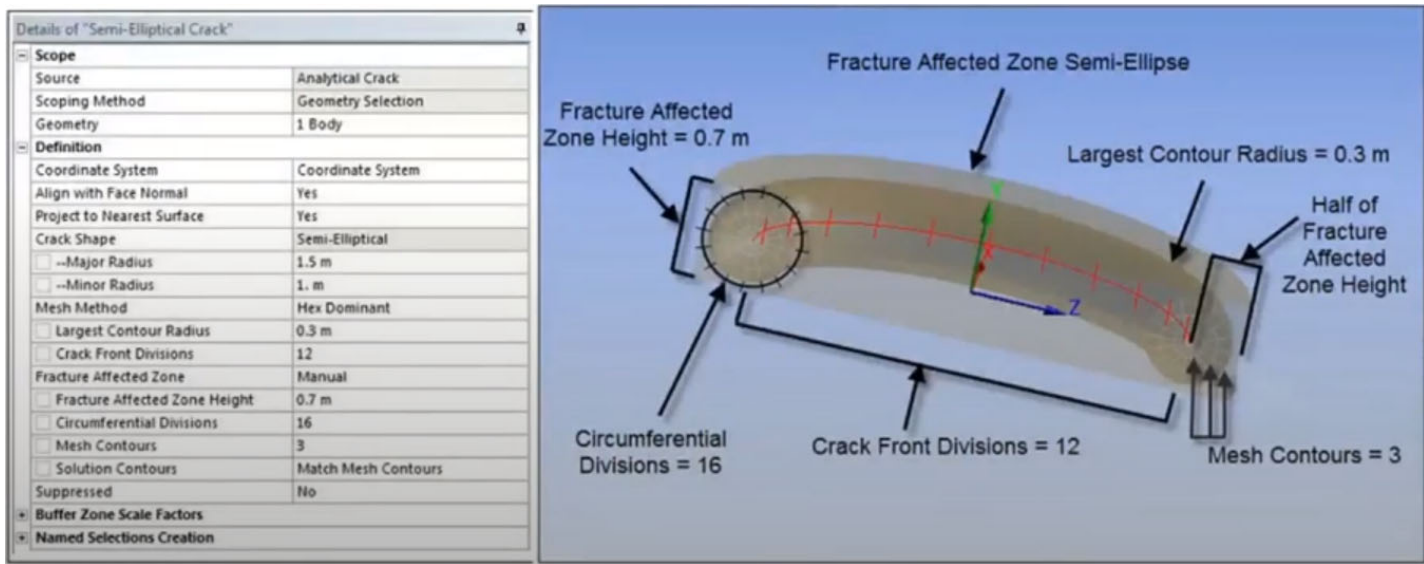

Figure 3. Properties of a semi-elliptical crack

For the crack, both hexa and tetra mesh are supported. The overall mesh of the selected body should only consist of tetra elements (Figure 4).
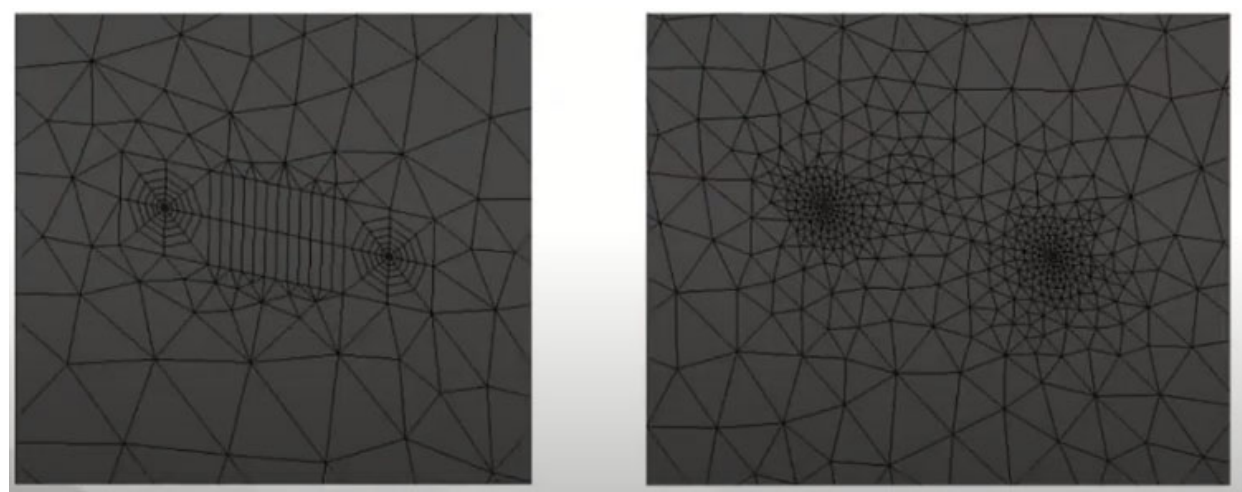

Figure 4. Example of Hex and Tetra Mesh for Semi-Elliptical Cracks 
The crack is applied to the selected surface according to the selected, pre-created coordinate system. In the case of a preliminary calculation of the strength without a crack, there is an option to create a coordinate system aligned along the principal stresses.

\subsection{Freeform Crack Arbitrary Crack}

The Arbitrary Crack tool allows you to simulate freeform cracks.

The base for the crack is the surface body created during the geometry step. An arbitrary crack also requires a mesh setup and a custom coordinate system. Only the creation of a tetra mesh in a crack is supported.

\subsection{Geometric Crack Pre-Meshed Crack}

The Pre-Meshed Crack object relies on a pre-created mesh with a crack, to define the front of which a special named nodal set is created.

In addition to the defining named set and the number of contours, the corresponding coordinate system is also specified in the settings. This is the only approach that supports a $2 \mathrm{D}$ solution.

The calculation of the fracture mechanics results is the same for any of the three instruments.

\subsection{Limitations in fracture mechanics calculations}

For objects, the Semi-Elliptical Crack Tool. Arbitrary Crack and Pre-Meshed Crack have several limitations:

- Fracture mechanics does not support adaptive mesh rebuilding (except SMART).

- For Semi-Elliptical Crack and Arbitrary Crack objects, only quadratic tetra mesh is supported as the base grid. Linear elements can be in the model, but at the most significant distance from the buffer zone of the body with Semi-Elliptical Crack and Arbitrary Crack objects.

- Only 3D calculations support Semi-Elliptical Crack and Arbitrary Crack objects.

- Semi-Elliptical Crack objects can be attached to one solid and the crack cannot propagate to more than one face.

- The stiffnes behavior to which the Semi-Elliptical Crack is attached / Arbitrary Crack must be set to flexible.

The Arbitrary Crack object can only be attached to one solid body. Only a single surface body can be selected as a crack surface, and this surface body cannot be embedded into a solid.

- Arbitrary Crack can only be broken with tetrahedrons.

- Calculation of fracture mechanics parameters based on VCCT is supported only for fracture meshes with lower order features. Therefore, VCCT-based fracture mechanics calculation is only supported for the Pre-Meshed Crack object.

\subsection{Fracture Modeling Tools}

The simulation of the crack propagation process is performed using one of the following approaches:

- $\quad$ VCCT-Based Interface Element Method

- Cohesive Zone Method

- SMART Crack-Growth Method

- XFEM-Based Method 


\subsection{VCCT-Based Interface Element Method and Cohesive Zone Method}

When the geometrical final path of crack propagation is known and specified in the problem, either the virtual fracture closure method (VCCT) or the bonding zone method (Cohesive Zone or CZM) can be used to simulate the edge separation process.

The Virtual Fracture Closure (VCCT) method was developed to calculate the rate of release of elastic energy, the critical value of which is the trigger for the crack propagation process. It is widely used to simulate phase separation in composite materials, but it can also be used to simulate the growth of cracks in metals.

The VCCT approach is based on fracture mechanics and therefore requires an initial crack (in PreMeshed Crack format) in the geometry.

The VCCT approach uses interface elements.

Bonding Method (CZM) establishes the relationship between crack opening and opening force at the interface. The method is also well used in calculating the delamination of a composite material, but it can also be useful in calculations with metals.

The constitutive ratios are set using a special material model in Engineering Data.

The approach itself is implemented using the Interface Delamination tool, as well as with the help of a separate Contact Debonding tool and does not require a preliminary crack object - only a geometric interface.

Both techniques rely on the assumption that the crack growth path is known and implemented in the geometric model.

\subsection{SMART Crack Growth}

Separating, Morphing, Adaptive and Remeshing Technology (SMART) is a computationally efficient fracture simulation method based on adaptive meshing technology.

Implemented using a separate SMART Crack Growth tool. It is assumed that the fracture mechanics branch and some original crack have already been added to the project. Semi-Elliptical Crack objects can work in this role. Arbitrary Crack and Pre-Meshed Crack.

This method affects the mesh only in the local area near the crack front and allows modelling both static and fatigue crack growth.

In the mode of calculating static crack growth, the process is controlled by one of two possible set criteria: the critical value of the stress intensity factor (fracture toughness) and the critical value $J$ of the integral. As soon as a critical value is reached at a given loading level, the crack begins to grow.

The crack grows either up to the specified limit, or to the point of impossibility of creating a new mesh, usually corresponding to the complete separation of the body into parts.

Despite the forced linearity of the problem, in most situations several substeps are required to simulate crack growth.

In the mode of calculating fatigue crack growth, the Paris law is used to simulate the process, which connects the rate of crack propagation under a cyclic load with the parameters of fracture mechanics.

In this mode, the applied load starts to be considered as a cyclic load with a constant amplitude, and the cycle asymmetry factor can be set in the properties of the SMART Crack Growth object.

In addition, there are two algorithms for modelling the process: calculate the number of cycles using a given crack increment, or calculate the crack increment based on a given number of cycles per substep.

\section{Solving the problem of modelling the process of crack propagation by the SMART method}

First, we create an object - a pipe $300 \mathrm{~mm}$ long, $48 \mathrm{~mm}$ in diameter and $6 \mathrm{~mm}$ thick. The crack profile is the intersection of the generatrix of the cylinder and the solid object (Figure 5). If there is a real crack size, then the correct shape and size of the defect can be drawn.

Next, in Static Structural - Mechanicals, set the properties for the crack object. For the crack object, set the Treatment parameter to Construction Body. So that it can be selected where we want to select 
it. To simulate the crack, we first created an additional coordinate system and set the dimensions of the crack using the "Semi-Elliptical Crack" command.

We build a $2 \mathrm{~mm}$ tetraid mesh. As for the three-dimensional grids, they use their own methods: Tetrahedrons, Sweep, Hex Dominant, MultiZone and Automatic. The Tetrahedrons method is suitable for our task. It allows generating volumetric meshes with tetrahedron-shaped elements based on one of two methods: Patch Conforming and Patch Independent (Algorithm section of the Tetrahedrons method).

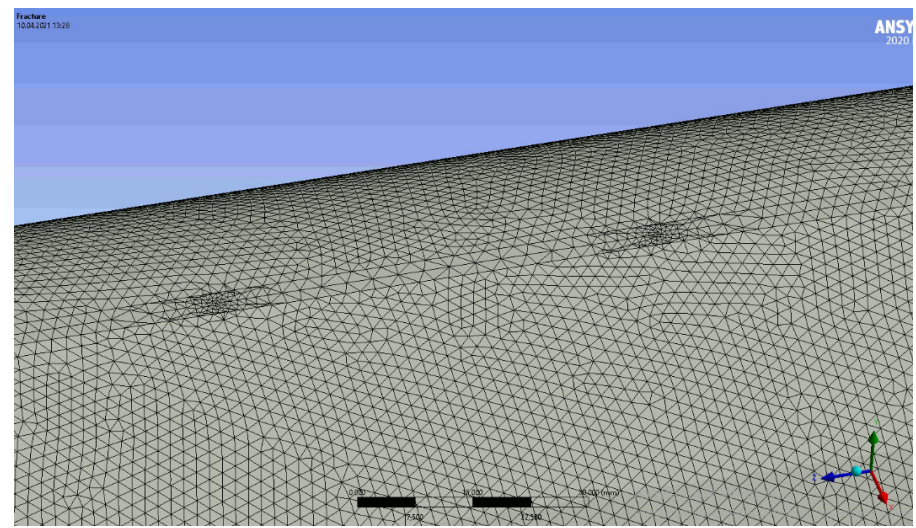

Figure 5. Crack profile creation

The crack profile in the pipe is shown in Figure 6.

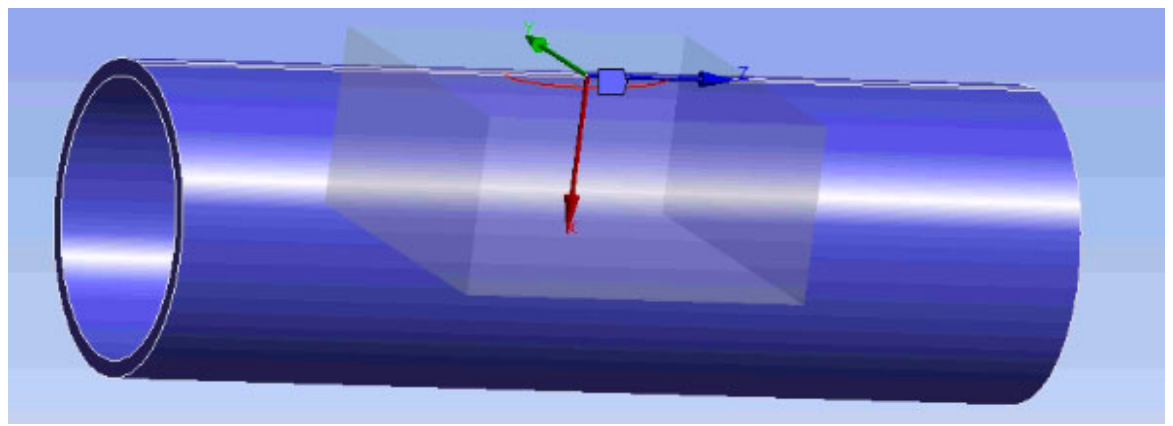

Figure 6. Profile of a crack in a solid object Stress

The simulation results are shown in Figures 7 - 8. Figure 7- total deformations. Figure 8 - Equivalent

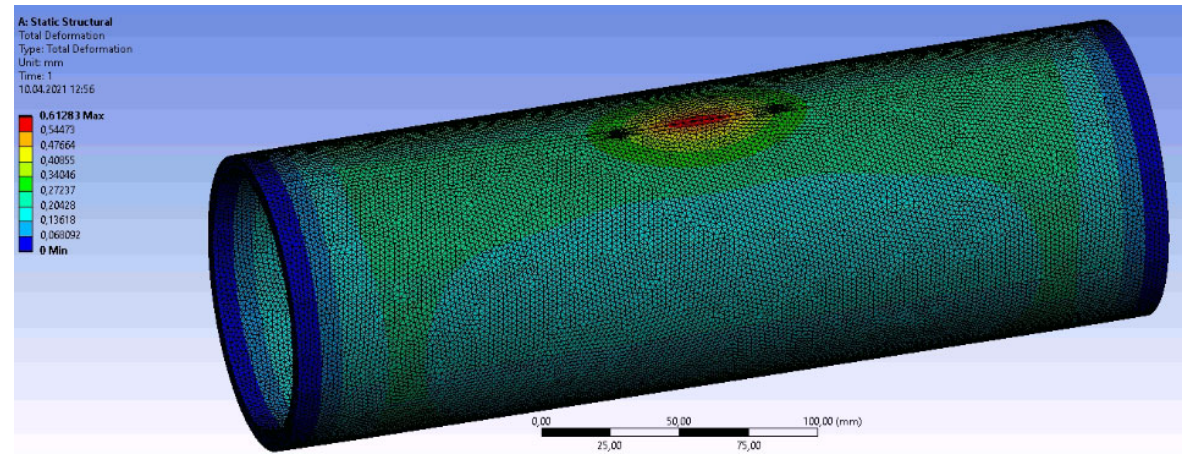

Figure 7. Profile of a crack in a solid object 


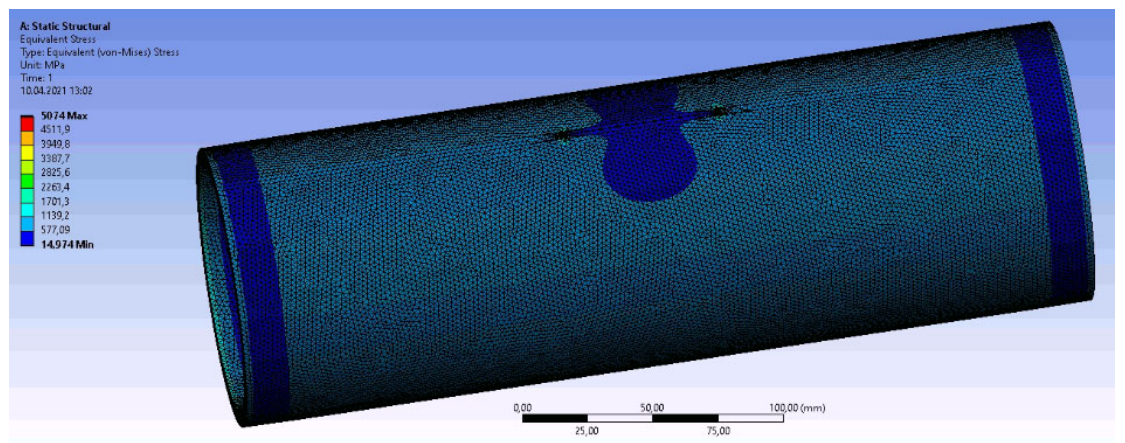

Figure 8. Equivalent Stress

Figure 9 shows a plot of fracture growth rate and strain versus pressure change inside the pipeline.

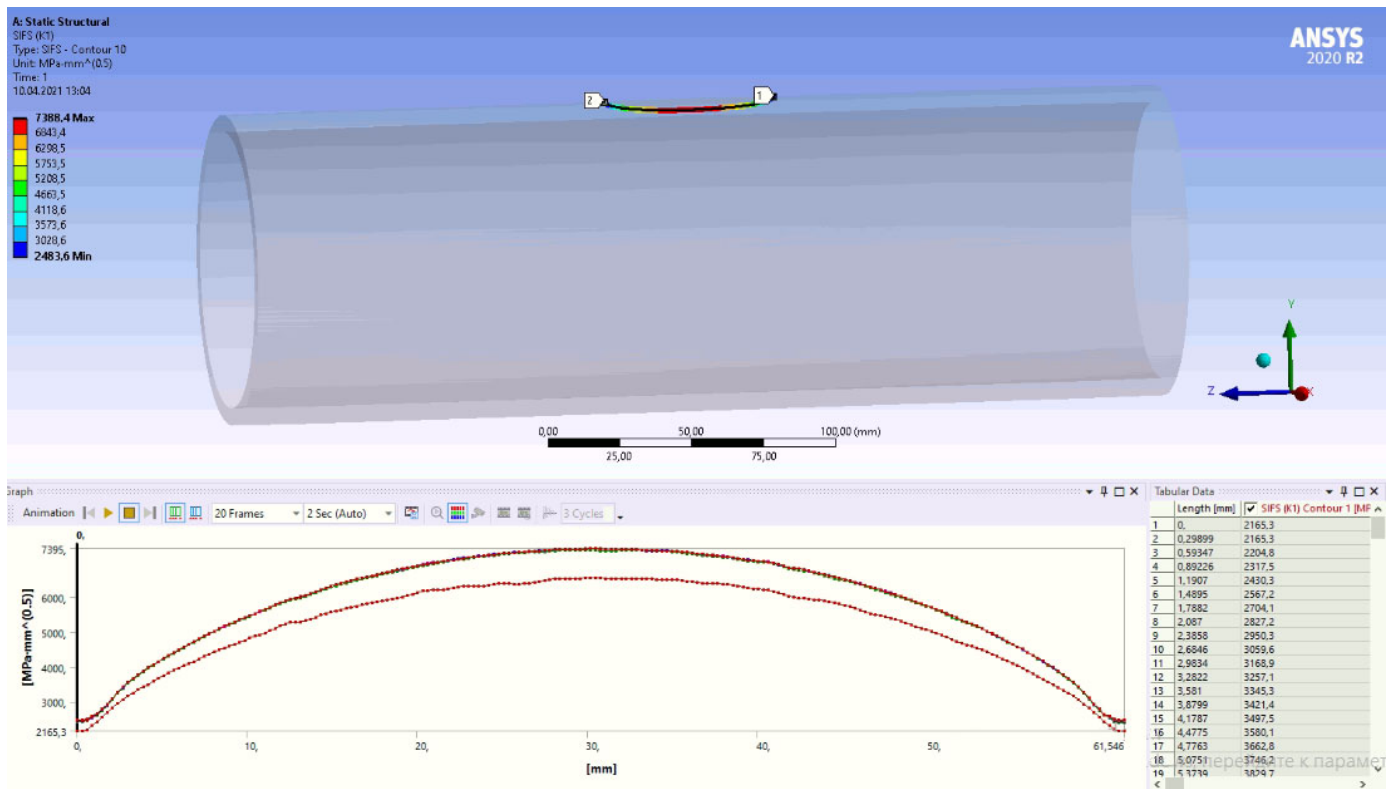

Figure 9. Fracture growth rate and strain versus pressure change inside the pipeline

\section{Conclusion}

Based on the results of modelling the stress-strain state of a fitting branch pipe with an extended linear defect $1=6 \mathrm{~mm}$ long, $\mathrm{h}=1 \mathrm{~mm}$ deep and $\mathrm{b}=0.1 \mathrm{~mm}$ wide of various shapes and different locations on the outer surface of the pipe, the following conclusions can be drawn:

- shows an algorithm for modelling the growth of an arbitrary-shaped crack Arbitary Crack under the stress-strain state of the pipeline, taking into account a linearly extended defect using J-integral - one of the algorithms for implementing fracture mechanics;

- it was proposed to use a nomogram to determine the maximum stresses in the zone of an extended linear defect with a length of $l=6 \mathrm{~mm}$, a depth of $\mathrm{h}=1 \mathrm{~mm}$ and a width of $b=0.1 \mathrm{~mm}$, depending on the pressure in the range from $1 \mathrm{MPa}$ to $25 \mathrm{MPa}$ at a temperature of $20^{\circ} \mathrm{C}$.

\section{References}

[1] A. Mishchenko, IOP Conference Series: Materials Science and Engineering 953(1) (2020) 012004.

[2] A. Sładkowski, Y. Proydak, V. Ruban, Transport Problems 15(3) (2020) 139-151.

[3] V. Vasylyshyn, I. Taras, I. Bekish, O. Kornuta, V. Kornuta, Management Systems in Production Engineering 28(2) (2020) 97-103.

[4] I. P. Aistov, K. A. Vansovich, Journal of Physics: Conference Series 1441(1) (2020) 012083. 
[5] A. Bambura, I. Mel'nyk, V. Bilozir, T. Prystavskyi, V. Partuta, Eastern-European Journal of Enterprise Technologies 1(7-103) (2020) 34-42.

[6] V. V. Harionovskij, Gas Industry Magazine 752(5) (2017) 56-61.

[7] A. S. Tyusenkov, Journal of Chemical Technology and Metallurgy 4(52) (2017) 766-772.

[8] R. N. Bakhtizin, F. M. Mustafin, L. I. Bykov, R. R. Khasanov, R. N. Kunafin, SOCAR Proceedings 3 (2016) 52-58.

[9] J. Abhilash, B. Apeksha Acharjee, IOP Conference Series: Materials Science and Engineering 455(1) (2018) 012113.

[10] S. Turbinsky, V. Urbanovich, V. Antonovich, Reviews on Advanced Materials Science 20(2) (2009) 136-142. 